PSICOLOGÍA

IBEROAMERICANA
Psicología Iberoamericana ISSN: 1405-0943

revista.psicologia@ibero.mx

Universidad Iberoamericana, Ciudad de México México

\title{
Análisis del superyó en un estudio de caso
}

Hernández López de la Fuente, Pamela

Análisis del superyó en un estudio de caso

Psicología Iberoamericana, vol. 26, núm. 2, 2018

Universidad lberoamericana, Ciudad de México, México

Disponible en: http://www.redalyc.org/articulo.oa?id=133959841008 


\title{
Análisis del superyó en un estudio de caso
}

\author{
ANALYSIS OF THE SUPEREGO IN A CASE STUDY \\ Pamela Hernández López de la Fuente \\ Universidad Latina de México, México \\ pamelahlf@outlook.com
}

Redalyc: http://www.redalyc.org/articulo.oa? $\mathrm{id}=133959841008$

Recepción: 08 Diciembre 2018 Aprobación: 05 Marzo 2019

\section{Resumen:}

En el presente artículo se realiza un estudio de caso desde el análisis de la instancia superyoica, revisado con un marco teórico psicoanalítico, se suma con ello material clínico que pudiera ser utilizado como referente en el estudio del superyó. Se hace una lectura desde las características observadas en un video de 1990 que documentó las entrevistas del tratamiento de Beth Thomas y el testimonio de sus padres adoptivos. Beth fue una niña de 6 años y medio, dada en adopción a los 19 meses, después de ser encontrada en condiciones paupérrimas tanto físicas como emocionales. Posteriormente presentó manifestaciones de agresión graves dirigidas no sólo hacia sus padres adoptivos y su hermano, sino también hacia ella misma, producto de un superyó cruel que buscaba el castigo. Se discuten de manera general los alcances del superyó de Beth en su relación con el diagnóstico psicoanalítico.

Palabras ClaVE: Superyó, agresión, sexualidad, maltrato, pulsión de muerte.

\section{Abstract:}

In this article, a case study is realized through the analysis of the super ego. The case study uses a psychoanalytic theoretical framework and additional clinical material. The reading is based on the characteristics of a video documented in 1990 about Beth Thomas's therapeutic treatment and also includes the testimony of her adoptive parents. Beth was a 6-and-a-half-year-old girl, who was given up for adoption at 19 months old. She experienced poor physical and emotional health, which manifested in serious aggression not only towards her adoptive parents and her brother, but also towards herself. This is as a result of the superego's needs to punish the self. The scope of Beth's superego is discussed in relation to her psychoanalytic diagnosis.

KEYWORDS: Superego, aggression, sexuality, abuse, death drive .

\section{INTRODUCCIÓN}

Para comenzar, dedicaré unas líneas para revisar de manera breve la concepción freudiana del superyó, pues fue ésa la base sobre la cual después se irían agregando diferentes aportes teóricos alrededor del concepto.

Cuando Freud expuso la génesis y funciones del superyó, retomó como primera aproximación sus observaciones de los delirios de observación en pacientes psicóticos y supuso que algo de eso debía existir en el resto de las personas. Atribuyó entonces al yo una parte heredera de la identificación parental y el complejo de Edipo, resultante en una instancia nueva llamada superyó (Freud, 1932).

Fueron tres las funciones que logró advertir: "Le hemos adjudicado la observación de sí, la conciencia moral y la función de ideal” (Freud, 1932, p. 62). Quedó con ello instalado en el pensamiento psicoanalítico el concepto del superyó como la instancia que vigila, castiga y orilla a seguir un legado parental.

Por su parte, Melanie Klein planteó otras posibilidades, pues en sus observaciones a niños muy pequeños, le fue inevitable apartarse poco a poco de esta concepción, sobre todo en cuanto al origen y los factores que se ven inmersos en su funcionamiento. Pues el legado freudiano la orillaba a un encuadre muy reducido que no se ajustaba a lo que su experiencia le dictaba. Klein sostuvo que los niños pequeños experimentaban culpa intensa, incluso mayor a la que experimenta un adulto, optando por delegarle al superyó un origen anterior al complejo edípico y reposicionándolo en sus inicios paralelo a la pulsión de muerte, como escisión del yo (Hinshelwood, 1989).

En el caso que expondré a continuación se halla la existencia de un superyó cruel, que busca el castigo como objetivo y trasfondo de su presencia. El eje del cual partiré para la lectura de tal cuestión son las aportaciones 
de Melanie Klein respecto de la concepción de dicha instancia psíquica, pues permite una comprensión más vasta del superyó, que alberga a los objetos internos interactuantes y la pulsión de muerte como referente. Esto, sin dejar de recurrir a otros autores para enriquecer el contenido del escrito.

Me pareció importante seleccionar un caso que me permitiera observar de manera menos impuesta la versión presentada al público, ya que en la actualidad es común encontrar en los medios de comunicación contenidos de violencia, y en general se suelen presentar los testimonios de las víctimas a detalle. Sin embargo los testimonios de los agresores casi siempre se presentan con una narración amarillista y sensacionalista que más allá de ser testimonio del agresor termina siendo la versión seleccionada del periodista o reportero que lo narra. Este video me resultó valioso, pues aunque no es presentado desde la mirada psicoanalítica, sí incluye la versión del sujeto, donde gracias a su formato permite la observación detenida de las palabras y manifestaciones pulsionales a través de la mirada, gestos y tonalidad de voz.

Aunque el material principal que analicé fue el discurso de la propia Beth, puesto que mi interés radica en la lectura de su realidad psíquica y no de la realidad objetiva, también tomé en cuenta ciertos datos mencionados tanto por los padres adoptivos como por el narrador del video, de tal forma que pude ampliar la visión del caso y abarcar otros puntos que consideré importantes.

Escribir sobre un caso desde la mirada psicoanalítica resulta muy vasto, y debido a las condiciones de tiempo y espacio para la escritura y publicación de este artículo, adecué este testimonio únicamente al análisis del superyó con los elementos que el video me permitió tomar y las limitantes y posibilidades que el material ofrece, con ello pretendo aportar al concepto del superyó evidencia que sirva como referente para nuevas concepciones o aportaciones.

Con el fin de apreciar con mayor detalle el discurso de los entrevistados, decidí citar ciertas frases del idioma original y corregí la traducción al español debido a ciertas imprecisiones de la traducción realizada para el video que resultaban relevantes para su interpretación.

\section{EL SUPERYÓ COMO ABANICO DE POSIBILIDADES}

Parte del eterno drama humano gira en torno a la instancia a la que llamamos superyó, en ella caben aspectos primitivos que nos acontecen a todos, que "como sedimentación de identificaciones primarias de la prehistoria personal y marca de la intrusión traumática de la pulsión en el viviente” (Juchnowicz, 2016, p. 358) se manifiesta como un entramado de revoluciones intra y extra psíquicas con resultados diversos, que sólo pueden ser palpados a detalle cuando son contados de manera individual.

En ocasiones, al referirnos a la instancia superyoica pareciera que hablamos de algo que está instalado en el sujeto como fiel representante de ciertas funciones palpables en el interactuar del individuo con el mundo externo, y entonces podemos quizá llegar a simplificar en la clínica al hablar de un superyó rígido con aquel que no se permite cometer pequeñas faltas, y lo contrario con aquel que no le importe recurrir a graves faltas, mientras le sea posible cometerlas. Pero no debiera ser ése el camino a seguir, pues

Cuando hablamos de ello, yo y superyó no se debe presuponer que constituyen entidades-cosas, sino conceptos para referirnos a conjuntos de actividades del psiquismo, que son en última instancia las que sí tienen existencia. Las agrupaciones son arbitrarias, podrían ser otras, y constituyen simples recortes de una totalidad (Bleichmar, 2016, p. 299).

En ese abanico de posibilidades, nos encontramos con aspectos que parecen ser polaridades distantes que, si bien terminan siendo las dos caras de una misma moneda, tendrán consecuencias muy distintas que estarán arraigadas a la naturaleza subjetiva de la psique de cada sujeto y en ocasiones apenas podrá ser evidente y palpable en su actuar. Klein (1958) plantea esta mancuerna cuando afirma que "la acción del superyó va desde la limitación de los impulsos destructivos, la protección del objeto bueno y la autocrítica, hasta las amenazas, quejas inhibitorias y persecución" (p. 245) y digo mancuerna refiriéndome a los resultados, a estos dos panoramas en los que el sujeto se puede encontrar y no, a la manera de dos caminos que se le presentan a la vez al 
sujeto, como si se tratara de la elección precisa del momento. Más bien podría metaforizar con una especie de recorrido en carretera en el que primero se encuentra el sujeto del lado de la mancuerna más tiránico, cruel y perseguidor, y si las condiciones lo permiten podrá llegar al otro extremo donde se encuentra finalmente el lado más amable del asunto.

\section{EL CASO DE BETH THOMAS}

Siguiendo el material de Monet (1990), Beth Thomas fue adoptada a los 19 meses, junto con su hermano Jonathan de 7 meses. Tim y Julie, una pareja conservadora estadounidense, quienes llevaban doce años de casados, al no poder tener hijos decidieron adoptar. Aunque Tim menciona no haber necesitado hijos para que sus vidas estuvieran completas, decidieron aventurarse a la adopción como medio para compartir y transmitir dicha completud; por su parte Julie parecía más entusiasmada ante la idea de la adopción, mencionó que aquello era un sueño compartido por ambos.

Finalmente en 1984 les dieron el aviso de la disponibilidad de adopción de Beth y Jonathan, se sorprendieron por la rapidez del trámite y se les dijo que eran normales y sanos, como si se tratase de niños sin pasado, como dos libros en blanco. Después obtuvieron información respecto a las condiciones de vida que tuvieron ambos niños antes de su adopción, siendo las condiciones de cuidado básicas, como alimentación e higiene, precarias y con evidencias de maltrato, donde incluso el desarrollo físico de los niños presentaba ya cierto deterioro.

Al poco tiempo notaron que Beth comenzaba a mostrar conductas de carácter agresivo y sexual que se iban incrementando poco a poco, principalmente dirigidas hacia su hermano, aunque también hacia sus padres adoptivos y animales domésticos; por medio de golpes, patadas, el uso de alfileres para picarlos, pellizcos en los genitales de su hermano, masturbación genital autolesiva en lugares públicos y confesiones sobre querer matar a Jonathan, Tim y Julie.

$\mathrm{Al}$ verse rebasados ante dicha situación, decidieron llevar a Beth con el Dr. Ken Magid, psicólogo clínico especializado en niños maltratados, quien llevó el curso de la entrevista con Beth presentada en el video, cuando ella tenía 6 años y medio de edad.

Antes de continuar con el siguiente apartado, en el cual haré referencia a eventos de la vida de Beth de manera más específica a la par del análisis de los mismos, es necesario apuntar que me resulta curioso e importante destacar el contraste que existe entre Tim y el padre biológico de Beth. De este último no se menciona gran cosa más allá de la evidencia de maltrato encontrada en los niños, sin embargo Tim es pastor de una iglesia, representante social del aspecto moral y bastante contrario al perfil que parece haber existido en el padre biológico.

\section{¿CÓMO HABLA EL SUPERYÓ DE BETH?}

Durante la entrevista se habla mucho sobre el papel del padre biológico de Beth, pues parece asumirse que fue el protagonista y culpable de la condición sintomática de Beth, tanto por las condiciones en las que fueron encontrados los niños, como por una pesadilla significativa que tuvo la niña; en la cual aparecen elementos de agresión por parte de su padre biológico hacia ella. Beth se sitúa en el sueño a la edad de un año, coincidente con el suceso de la muerte de su madre, sin embargo, en el video se menciona sólo en una ocasión a la madre biológica, para decir sin más detalle que murió.

No se menciona el trato de la madre biológica hacia sus hijos ni las consecuencias de su muerte tanto en los hijos como en el padre de ellos, pero ante dichas condiciones es posible que Beth se viera envuelta en una suma de circunstancias que la llevasen por los caminos posteriores de agresividad, como refiere Levisky (1995) "a través de actitudes antisociales hacen su apelo inconsciente, como manifestaciones del abandono, 
de la carencia, de la privación" (parr. 6). Resulta indudable que esa pérdida temprana de objeto constituyó un primer pilar básico que fundamenta la vulnerabilidad posterior de Beth, enfrentada después a un padre que se iría también, anunciándose así como una segunda pérdida: no sólo la abandona, además la amedrenta, sumándose como segundo pilar en su particular construcción superyoica.

Grinberg (1983) menciona que ante la pérdida temprana de objeto, además de generar culpa, "en lugar de incorporar al objeto perdido, como suele ocurrir en el duelo normal, estos niños tendían a proyectarlo en el mundo externo con rabia y desilusión. Su conducta antisocial era una forma de atacar al objeto afuera como una especie de venganza y de control” (p. 100). Pero ¿̇venganza y control dirigidos a quién? La evidencia clínica en Beth, como revisaré más adelante, sugiere que ella no sólo atacaba al objeto sino también a sí misma. Es este mismo autor quien señaló que ante el inevitable ataque en la fantasía del objeto y frente a su pérdida, el sujeto se inunda de culpa, pues se atañe responsabilidad de dicha pérdida objetal (Grinberg, 1983). En mi interpretación, Beth se enfrentaba a ataques no sólo dirigidos hacia el exterior sin más, sino que se hallaba involucrada en dichos ataques, su yo fue responsable inevitablemente y el otro depositario de sus propios aspectos disociados.

Fue Klein (1958) quien atribuyó a la formación del superyó la proyección desde el yo de los objetos previamente internalizados tanto buenos como malos, ligando los primeros a una madre que alimenta y los últimos a una madre que frustra; estos dos elementos constituyen aspectos importantes de las funciones superyoicas adecuadas u óptimas, que dependerán de la integración conciliadora de ambas, ¿̨puede decirse que existen elementos cuantitativos respecto a lo bueno y lo malo?

Tim menciona a propósito de los antecedentes de Beth: "descubrimos que no tenían suficiente comida, que quizás incluso Beth pasaba todo el día a lo mejor con tan sólo una caja de cereal” (Monet, 1990). Sumado a ello, en la pesadilla mencionada, Beth comenta al respecto "cuando él tocó mi vagina hasta que sangrara, dolió mucho hasta que sangraba, no me alimentaba mucho" (Monet, 1990). Lo que resulta interesante es que, al haberse soñado a la edad de 1 año, época en la que murió su madre, el aspecto de la alimentación aparece entonces como proveniente del padre biológico.

En ambos discursos, el de Tim y Beth, aparece el elemento cuantitativo referente a la alimentación, como si se tratara de que aquello que se dio de alimento para mantenerla con vida hubiese sido meramente por supervivencia, pero no suficiente para el alimento psíquico, tal como refiere Levisky (1995):

Del encuentro del bebé con su madre, a partir de su conjunto de elementos innatos y potenciales, dentro de condiciones emocionales y existenciales adecuadas, suficientemente buenas, el bebé establece con su cuerpo, con la madre y posteriormente con el padre, representantes del primer conjunto social, una relación creativa, de desarrollo de sí mismo. Estas condiciones son fundamentales para el proceso de identificación, formación de las primeras identidades y del superyo (párr. 3).

Se despliega nuevamente contenido valioso de la pesadilla de Beth, pues en palabras de Tim puedo notar la presencia de objetos parciales, cuando refiere que "la pesadilla era sobre un hombre que se caía sobre ella y la lastimaba con una parte de sí mismo" (Monet, 1990). No quedan claros los límites de "una parte de sí mismo" al referirse, ambiguamente, a aquello con lo que fue lastimada, como si estuviera construida la relación con objetos parciales.

Se suma una relación mano-vagina parcializada con dos sucesos clave, el primero de ellos es un dibujo realizado por Beth mostrado al entrevistador, donde aparece su padre biológico tocando su vagina, el cuerpo del padre y el torso de ella dibujados en color azul, pero la mano de él y la parte inferior del cuerpo de ella dibujados en verde como en una especie de fusión. Al preguntarle por la ubicación de la mano, ella le responde "justo ahí, no puedes en verdad verla porque es verde" (Monet, 1990). El segundo suceso es referente a que Beth se masturbaba diariamente hasta causarse daño, menciona "lo hice todos los días hasta que se puso muy mal y me detuve y tuve que ir al médico y no se veía bien” (Monet, 1990). Únicamente cesó cuando por fin llegó a tal estado, donde incluso sangraba, además afirmó que tenía "gérmenes y cosas de mi mano" (Monet, 
1990). Las “cosas de su mano" se fusionan con su vagina, al igual que la mano de su padre biológico dibujada sobre su zona genital.

Para Beth fue necesario masturbarse diariamente, se agregan como una constante las agresiones dirigidas principalmente a su hermano Jon, donde ella aseguraba no poder parar, a pesar de las súplicas de él (Monet, 1990). ¿Por qué aquello que resultó tan doloroso busca repetirse? ¿Por qué Beth no puede parar?

$\mathrm{Al}$ avanzar en su obra, Freud llegó al punto en el que la pulsión sexual quedaba ya no como la única, sino que a partir de ella se desprendían la pulsión de vida y la pulsión de muerte (ubicada en las profundidades del ello), y fue para el año de 1920, cuando en Más allá del principio del placer comenzó a dudar acerca de los alcances aparentemente absolutos en la psique humana del llamado principio de placer. Esta inquietud parece surgir a partir de la obsesión de repetición observada en los repetidos sueños de los afectados por neurosis traumática y en el juego infantil igualmente repetitivo; parecían escapar de una explicación lógica en la que pudiera tener cabida la obtención de placer (Freud, 1920). Así mismo, Beth se encontraba sumergida, a partir del evento traumático, en la compulsión a la repetición que le permitía asimilar el trauma (sin ser sinónimo de curar), ya que como mencionó Laplanche (1984):

La compulsión de repetición está ligada esencialmente a la pulsión de muerte, y que todo lo que es progreso, tanto en la simbolización como en el duelo, y de un modo general lo que introduce cambios en la cura, está ligado esencialmente con la pulsión de vida (p. 124).

Siguiendo la misma línea, surge otro aspecto interesante en Beth respecto al contenido de lo que buscaba asimilar, y como dejaba entrever aspectos que resultan paradójicos a simple vista, pues vemos que se articulan aspectos que pertenecen a un superyó, que se hizo presente de manera un tanto engañosa, pues Beth se hacía atrapar a través de un "superyó mudo y feroz producto de la no inclusión en la ley del padre, que deja detenido al sujeto en el lugar en el cual, mediante la transgresión, se llama a Otro” (Breu, Yosco, \& Ricardo, 2012, p. 253).

Me parece que el suceso más evidente de mi anterior afirmación fue cuando Beth robó a su madre adoptiva unos cuchillos de la cocina, confesando que era su intención matar a su hermano y padres adoptivos. Julie sospechaba de ella, sin embargo, prefirió callar para descubrir semanas después que, sin haberlos utilizado, Beth se pusiera en total evidencia al preguntar a Julie por el aspecto de sus cuchillos perdidos, como mostrando discretamente el robo cometido después de que Julie lo hiciera pasar inadvertido. Después de haber hecho la pregunta que la delataría, dejó escapar una sonrisa; al respecto Julie comenta "y luego esa pequeña sonrisa que no es una sonrisa dulce, sino un tipo de sonrisa maliciosa" (Monet, 1990).

Cuando se tocó el tema de la masturbación de Beth, Julie narró que aunque previamente ella ya le había explicado que ésas eran áreas privadas y no debía hacerlo en lugares públicos, Beth se masturbaba en presencia de su madre, en una ocasión en un estacionamiento público. En otra ocasión, Beth descubrió un nido con crías de pájaro, Julie le advirtió que debía dejarlas, pues las podía lastimar. Beth parece haber capturado otro detalle de la advertencia de su madre cuando escuchó "que la madre no regresaría si alguien tocaba a sus bebés" (Monet, 1990). Como si algo hubiese resonado en su propia historia y ésa fuera la ocasión perfecta para recrearla, hizo caso omiso de la advertencia, matando a las crías para, posteriormente, ser descrubierta. Mencionó que tomó a las crías para llevárselas a Julie y preguntarle si estaban muertas, luego relató que Julie llamó a Tim por medio de un grito. Es preciso hacer notar que justo después de haberse referido a la acusación de Julie, indudablemente dibujó una sonrisa en su rostro (Monet, 1990).

A través de maniobras para engañarse a sí misma respecto a sus intenciones, Beth buscaba el castigo sin poder escapar de la culpa de un superyó que le reclamaba y la incitaba a escenificar un crimen que quizá se cometió en la fantasía tiempo atrás, como un deseo parricida intenso vivido por las frustraciones cometidas en su contra. Laplanche (1984) sugirió que, si de algo no puede escapar un sujeto es de la angustia misma, donde a manera de despliegue se encuentra la culpa, una ramificación más de la angustia, donde quizá el crimen edí- 
pico ha de cometerse como consecuencia de la culpa del crimen previamente fantaseado, saboreado como tatuaje de la especie humana del que no se podría escapar.

Irremediablemente Beth ya había hecho suya esa misma ferocidad parental que le fue depositada por medio de identificaciones proyectivas por parte de su padre biológico y quizá también de su madre biológica. Beth no encontró otra salida más que seguir un camino similar del que fue receptora en aquel primer año de vida "por contraidentificación, buscando, a su vez, otros objetos en quienes descargar masivamente sus respectivas identificaciones proyectivas" (Grinberg, 1983, p. 105).

Para finalizar, como referencia a aquellas sonrisas mencionadas en párrafos anteriores y en concordancia con lo mencionado por Lieberman (2005):

Donde lo normal sería un grito, se hace un llamado. Pero el del humor no es cualquier llamado. No es un llamado de auxilio ya que no espera que llegue alguien a responder. El humor resulta bondadoso porque ha renunciado a toda esperanza (p. 173).

Es a través de la observación del video y del comentario que hizo Julie del suceso de Beth al ponerse en evidencia respecto al robo de sus cuchillos, que Beth mostraba en recurridas ocasiones alguna "sonrisita", reflejo de un superyó que hace un llamado a través del humor, las sonrisas como vía irremediable para apaciguar su debilitado yo, recurriendo a éstas como defensa ante su ya instalada vulnerabilidad.

\section{CONCLUSIÓN Y DISCUSIÓN}

Desde el relato específico e individual de Beth, podemos pensar en la existencia de un superyó sumamente agresivo y cruel, que se deja atrapar ante la demanda de castigo. La posiciona en una inclinación destructiva, custodiada por la pulsión de muerte, que amenaza de manera latente desde la compulsión a la repetición.

Si bien las concepciones teóricas del superyó difieren entre los distintos pioneros que han hecho escuela en psicoanálisis, mi contribución adquiere importancia principalmente desde la evidencia clínica, como campo fértil para contribuciones teóricas posteriores, que permitan hilar el legado psicoanalítico referente al campo de la instancia aquí revisada.

En el recorrido de este estudio de caso me encontré con ciertas limitaciones, por un lado se basa en lo que el material del video ofrece, por lo que se reduce a lo que el entrevistador posibilitó y deja de lado ciertas herramientas como el juego, que habrían brindado mayor alcance y riqueza a mis interpretaciones. Por otro lado, al ser mi objetivo el análisis del superyó de Beth, quedó relegada la totalidad que se pretende desde una metapsicología. Es indudable que no podemos reducir el análisis de un caso a una instancia psíquica y que quedan pendientes en el caso de Beth Thomas muchos otros aspectos por ser abordados.

A partir del soporte teórico que sirvió para argumentar mis observaciones del caso, surgió inevitablemente como interrogante a lo largo del estudio el aspecto diagnóstico, ¿se trata de una psicosis?, ¿ podemos hablar en términos de psicopatía o personalidad antisocial?, ¿en qué cambiaría el análisis del superyó de Beth al llevar en paralelo un diagnóstico? Con la conjunción de estos dos aspectos, podríamos acercarnos a una interesante precisión, que implicaría una elaboración más amplia para futuras investigaciones.

Desde la idea freudiana del superyó como heredero del complejo de Edipo, que parece tener sentido en las neurosis, las piezas no encajan en el caso de las psicosis, como se interrogan Rossetti y Perak (2017, p. 723):

Al definir Freud al superyó como heredero del Complejo de Edipo y resultado de la identiicación al padre, nos es lícito interrogar su estatuto en la psicosis, ya que dicha instancia pareciera, desde este punto de vista, quedar limitada sólo a las neurosis.

Freud no consiguió avanzar mucho en el campo de las psicosis, y aunque logró aterrizar sus concepciones del superyó, gracias a sus observaciones de ciertos psicóticos, no dejó en claro si existe la posibilidad de una ausencia o déficit superyoico en algunos sujetos, pues al tratarse de una instancia que engloba consigo ciertas funciones, ¿qué sucede cuando éstas parecieran no presentarse? 
En todo caso, Freud fue muy claro tanto en el abordaje amplio que merecen cada una de las instancias psíquicas, como en el peso que se debe otorgar al relato individual, al decir que

\begin{abstract}
No deben concebir esta separación de la personalidad en un yo, un superyó y un ello deslindada por fronteras tajantes, como las que se han trazado artificialmente en la geografía política. No podemos dar razón de la peculiaridad de lo psíquico mediante contornos lineales como en el dibujo o la pintura primitiva; más bien, mediante campos coloreados que se pierden unos en otros, según hacen los pintores modernos. Tras haber separado, tenemos que hacer converger de nuevo lo separado. No juzguen con demasiada dureza este primer intento de volver intuible lo psíquico, tan difícil de aprehender. Es muy probable que la configuración de estas separaciones experimente grandes variaciones en diversas personas, y es posible que hasta se alteren en el curso de la función e involucionen temporariamente (Freud, 1932, p. 74).
\end{abstract}

Aunque el aspecto diagnóstico no fue el objetivo de mi investigación, quedará como inquietud para ser revisada posteriormente, pues como menciona Sierra-Rubio (2014) pudiera ser incluso una manera de abordar casos que quedan relegados a denominaciones clínicas novedosas que conducen a un bagaje teórico que merece la pena ser replanteado.

\title{
REFERENCIAS
}

Bleichmar, H. (2016). Avances en psicoterapia psicoanalitica: Hacia una técnica de intervenciones especificas. Barcelona, España: Paidós.

Breu, R., Yosco, G., \& Ricardo, L. (2012). Estudio exploratorio sobre la relación entre superyó y acto delictivo en internos condenados reiterantes. Efectos de una ferocidad muda. En IV Congreso Internacional de Investigación y Práctica Profesional en Psicología XIX Jornadas de Investigación VIII Encuentro de Investigadores en Psicología del mercosur. Congreso llevado a cabo en Buenos Aires, Argentina.

Freud, S. (1920). Más allá del principio del placer (vol. cx). Madrid, España: Biblioteca Nueva.

(1932). $31^{\circ}$ conferencia. La descomposición de la personalidad psíquica. En Obras Completas: Nuevas conferencias de introducción al psicoanálisis, y otras obras (1932-1936). Buenos Aires, Argentina: Amorrortu.

Grinberg, L. (1983). Culpa y depresión: Estudio psicoanalitico. Madrid, España: Alianza Editorial.

Hinshelwood, R. D. (1989). Diccionario del pensamiento Kleiniano. Buenos Aires, Argentina: Amorrortu.

Juchnowicz, M. (2016). Carácter y superyó. Dos declinaciones posibles de la alteración del yo. En VIII Congreso Internacional de Investigación y Práctica Profesional en Psicología XXIII Jornadas de Investigación XII Encuentro de Investigadores en Psicología del mercosur. Congreso llevado a cabo en Buenos Aires, Argentina.

Klein, M. (1958). Sobre el desarrollo del funcionamiento mental. Obras completas (vol. 3). México: Paidós.

Laplanche, J. (1984). Reparación y retribución penales. Una perspectiva psicoanalítica. Revista Trabajo del Psicoanálisis, 3, 46-61.

Levisky, D. L. (Noviembre, 1995). Moral, superyo, delincuencia y democacia. En IV Encuentro Winnicottiano. Congreso llevado a cabo en Santiago, Chile.

Lieberman, M. (2005). Entre la angustia y la risa. México: UAM.

Monet, G. (Productor), \& Cohn, A. (Director). (1990). Child of rage [Niño de furia] [DVD]. EU: Home box office. De https://www.youtube.com/watch?v=oksKt21-jaY\&t=220s

Rossetti, A., \& Perak, M. (2017). Reflexiones acerca de superyó en la psicosis. En IX Congreso Internacional de Investigación y Práctica Profesional en Psicología XXIV Jornadas de Investigación XIII Encuentro de Investigadores en Psicología del mercosur. Congreso llevado a cabo en Buenos Aires, Argentina.

Sierra-Rubio, M. A. (2014). Sobre el status de la psicopatía en psicoanálisis. Boletín Cientifico Sapiens Research, 4(1), $21-25$. 